\title{
Information System Development in Resource Constraint Setting: A Case Study from Malawi
}

\author{
Martin Bright Msendema ${ }^{1}$,Patrick Albert Chikumba ${ }^{2}$ \\ mmsendema@poly.ac.mw ${ }^{1}$,pchikumba@poly.ac.mw ${ }^{2}$ \\ Faculty of Applied Sciences, University of Malawi - The Polytechnic, Malawi
}

\begin{abstract}
Information Communication Technology (ICT) has in so many ways proved to be the drive of economy and a game changer to many disciplines. However digital divide has been of concern as developed countries have been benefiting more than their developing counterparts. All that resulting from lack of skilled labour and finance to invest into the required technology. This case study focused on the survival of the developing countries as they are constrained by the financial challenges. Three major dimensions from the socio-technical perspective of systems dimensions guided the authors in the study. The study showed the ability of people in developing countries to adapt to the challenging environment as they strive to cultivate from the strengths of ICT. This is particularly within Small and Medium Enterprises. Primary to this adaptation is the use of local expertise to develop own system than relying on imports from developed countries.
\end{abstract}

Keywords: Information System, Information System Development, Social System, System Development Life Cycle, Resource, Resource Constraint.

\section{Introduction}

In the past few years, small and medium organisations in Malawi have realised the importance of ICT in improving their operations. However, due to economic constraints that the country is facing, as in other developing countries, these organisations are opting for local technical resources, including expertise, in their development of Information Systems (ISs). It has been noted that developing IS using local expertise, particularly application software, may increase the chances that it fits the context, culture, and language where it is used. Even UNCTAD recommends to governments in adopting policies that help build domestic software capabilities[1]. The creation of a local software industry indeveloping countries is seen as a potential catalyst for socio-economic development [2]. However, many organisations in developing countries have challenges in the development of IS due to factors such as lack of adequate skilled labour, economic constraints, systems infrastructure deficiency, and constraints imposed by the social and political contexts. Therefore, the aim of this study is to understand how ISs are developed in a resource-constraint setting, with Malawi as an example, from the socio-technical perspective. Three cases are used: (i) one Public Institution - focusing on Client Information Management, (ii) one Non-governmental Organisation (NGO) - focusing on monitoring and evaluation, and (iii) six Small Medium Enterprises (SMEs) - focusing on accounting and finance. Details on how and why these were chosen are presented Section 3.

In this paper, resources are considered as assets and capabilities that are available and useful in carrying out IS development activities. Capabilities and assets are respectively what 
an organization has and does [3]. Resources for an activity can be principal, dependent and independent resources. According to [4], a principal resource is the main resource whose usage amount may be depended upon by other resources to process the activity; a dependent resource is a resource whose usage quantity depends on that of its principal resource; whereas an independent resource is independent from other resources.

The term constraints can mean those things that limit person's options in carrying out activities. Resource constraints refer to the limited availability of resources that are necessary to complete an activity. Despite their disadvantages, resource limitations can result in bringing positive outcomes such as higher efficiency and encouraging innovation, that is, organisations may be forced to become more innovative, creative and resourceful [5].

\section{Information system development}

Researchers have discussed benefits of adopting ICT in organizations in developing countries in order to manage information. ICT is takenas any technology that enhances the means of storing, processing, distributing and exchanging information within an organisation and with its external environment. Due to ICT, distance and time are no longer an issue in accessing required information flows; ICT has improved coordination of activities within and beyond organisational boundaries [6]. However, the adoption of ICT is influenced by the social context in which it will be used. The social contexts and technological artefacts perpetually interact and shape each other [7]. Hence, this research considers both technical and social elements that together form ISs. ISs are influenced by environment, infrastructure, socio-cultural, and economic issues [8]. An IS is taken as a set of interrelated components (technical and non-technical) and organised procedures that collect, retrieve, process, store, and disseminate information to support decision making, control, analysis and visualisation in an organisation[9]. In ISs, people and organizationsutilize technologies in the management of information. IS are an essential component of organisational management and ICT is part of human life since the collective human activities make ICT appropriate. ISs provide users with specific information within organisational context, with the support of ICT [10].

From the brief discussion above, it can be observed that any IS consists of humans, organisation and technology. Hence, scholars refer to ISs as social systems. ISs exist in a real world consisting of objects, people, rules, norms, and commands [2]. ICT is just one aspect of ISs. ISsaredependent of people because their individual motivations, practices, values and interest influence their use of ICT. Apart from the benefits that ICTs bring to organisations, "they also raise the challenge of substantial organisational and human change" [11]. For better understanding of IS development, implementation and use, it is important to analyse social practices and organisational culture in which people are engaged [12].

Three important perspectives that help to understand ISs are human, organisation and technology, which are related, and shape each other[13]. The human perspective highlights various needs of individuals that lead to use of ICTs to perform their duties. People favour ISs that support their interests and dis favour those which threaten their interests [11]. From the organisation perspective, organisations compose of structures, goals, policies, and unique culture, among others that influence how ISs are used. ISs also use technologies to provide an effective and efficient way of processing data and transforming it into a variety of information products. This paper has used people, technology and organisation as three important dimensions of ISto discuss opportunities and challenges of IS development.

IS development is a critical part of the diffusion and implementation of ICT in a particular organisational context, and as a process, it involves various activities such as analysis, design, 
implementation, support provision, project management, quality assurance, and software process improvement [2]. IS development deals with adjusting and integrating pieces of software to fit the needs of a specific work activity, which requires activities and relations of different groups of stakeholders including users, professionals, management, academics and users' clients [2]. System development can be taken as the entire set of activities needed to construct an IS solution to a business problem or opportunity [14]. For a successful IS development, a development team needs some organised collection of concepts, beliefs, values and normative principles supported by materials, which is referred to as an IS development methodology [2]. However, various models for IS development exist and "need to be adjusted to any given socio-economic, cultural and organisational setting" [2].System developers need to remember that they are guided by influences related with the object of their work and the nature of their organisation, and how it affects expectations [10]. The traditional system development method used by most organisations is the System Development Life Cycle (SDLC). SDLC consists of sequential processes by which IS are developed - including system investigation, analysis, design, programming, testing, implementation, operation, and maintenance[10, 14].

SDLC can be in two generic types: The Waterfall Model and the Incremental Model [10]. The Waterfall Model involves successive stages sequenced downward like the flow of a waterfall and its emphasis is on the process, rigorous documentation, and self-contained stages and a bit of iteration. The Incremental Model involves successive builds or increments and with each build, the system is designed and developed; then the working version is implemented and tested by users within working contexts. The users provide valuable feedback, which will then be used as a starting point for the next build. Researchers have built ideal models of SDLC, which can be considered variants of the Waterfall Model, Incremental Model or combination. These may be options to complement or replace portions of SDLC in order to reduce development time, complexity and cost, which are some disadvantages of SDLC[14].

\section{Research methods}

This research was conducted in Malawifrom 2015 to 2016 (about 24 months) in three different organisations, which were purposively sampled and named as Organisation A (a Public Institution - Statutory Corporation), Organisation B (Non-governmental Organisation), and Organisation C (Small and Medium Enterprises). Organisation A was chosen as one example of public institutions, which has utilised its local resources to develop and implement IS for its client information management. This Statutory Corporation provides public services to both urban and rural disadvantaged communities across the country. The second author participated in the process of IS development for Organisation A. Organisation B is responsible for child nutrition projects across the country. It developed and implemented M\&E system using local technical expertise. Organisation B was chosen because it provided an access during the data collection as compared to other similar NGOs that were approached. Organisation $\mathrm{C}$ is a set of SMEs that have purchased and used a locally developed generic finance and accounting package by local software developers. The authors visited six SMEs in Blantyre, which have been using this package. In the time of the study, most SMEs using the package were in Blantyre city.

This is a case study in which qualitative interpretive methods were adopted, and multiple data sources were used. Taking IS as a social system, it is required to understand its context and the process whereby it influenced and was influenced by the context. Data was collected 
through interviews, participant observations and artefact examination. The interviews and artefact examination were conducted in all three settings within the period of study whereas the participant observation was only applied in Organisation A since the commencement of its IS development in 2010. In all three cases, semi-structured interviews with developerswere conducted first, then artefact examinations and finished with semi-structured interviews with users.

Sixteen semi-structured interviews were conducted with various participants (see Table 1). Each interview was conducted at interviewee's workplace. The interviews concentrated on the experiences; particularly approaches applied, technologies used and challenges faced. During the interviews with developers, critical functions of systems were demonstrated to the authors in order to appreciate on what the systems do.

Table 1.Semi-structured interviews with the participants

\begin{tabular}{|c|c|c|}
\hline Case & Total & Explanation \\
\hline Organisation $\mathrm{A}$ & 5 & $\begin{array}{l}\text { Two IT officers who provide technical support to users and were also } \\
\text { developers of the information system } \\
\text { Three managers who are users of the information system }\end{array}$ \\
\hline Organisation B & 3 & $\begin{array}{l}\text { Two developers of the information system } \\
\text { One user representative in the project }\end{array}$ \\
\hline Organisation $\mathrm{C}$ & 8 & $\begin{array}{l}\text { Two developers of the finance and accounting package } \\
\text { Six users of the package (one from each of six SMEs) }\end{array}$ \\
\hline
\end{tabular}

Participant observations were done in Organisation A by the second author as one of the developers and implementers. He participated as a volunteer in development and implementation activities including requirements analysis, design, coding, testing, documentation, deployment, user training, and post-implementation evaluations. The second author was the key technical personnel in the project and provided technical advice. His observations during the development of IS have contributed to the findings of this research.

The authors also used artefact examination to gather data from three organisations. Artefacts examined in this research include mainly data collection forms, operational manuals, databases, user interfaces, and generated reports. The system functions were analysed during the system demonstrations. The artefact examination helped the authors to understand the system designs and features.

Data analysis was done manually through four key steps: immersion in the data, coding, creating categories, and the identification of themes [15] being guided by the dimensions of socio-technical system. The authors moved back and forth through the four processes in order to make sense out of the whole dataset. The authors started the data analysis at the time of interviews and participant observations and this allowed the authors to identify problems and concepts that appear likely to help in understanding the situation.

\section{Findings}

\subsection{Organisation A (public institution)}

The computerised IS for Organisation A is web-based and developed by the second author as a volunteer with the support of ICT unit of the organisation. It was initiated by ICT unit in 2010 with three pilot districts and later it was rolled out to other districts. The IS was introduced in order to enhance storage, accessibility and reporting of data. ICT unit provides 
basic technical support to the systemusers. One ICT officer is the systemadministrator and facilitates user trainings. Organisation A has offices in all twenty-eight districts managed by district managers. Individuals, who need services from Organisation A, are registered by volunteers within communities and assessed by the district offices using paper forms. Each form is duplicated and its second copy is sent to the Head Office (ICT unit) for capturing into the system. Due to logistical challenges of sending paper forms, Organisation A is planning to decentralize the data entry to the district offices within the custody of their respective district managers. Only registered users from the Head Office and district offices access the system. If the user is within the Head Office, he/she accesses the system through the local area network (LAN); otherwise, Internet connection is required. The public can also access the system through the guest account but with limited privileges since this system contains personal data. Users with some specific privileges are able to view 'sensitive' reports and in some cases they can download them as Excel file, word file, or pdf.

The IS development is a two-tier architectureincluding data layer and business logic (plus interfaces). PHP and MySQLwere usedfor the business logic and database respectively. The system is running on Linux server and accessed through the Internet. As illustrated in, The server is hosted at the Head Office (see Figure 1). The Linux server was built on an ordinary desktop computer with a private (local) IP address. An Internet Service Provider (ISP), who provides the Internet service to Organisation A, is the one mapping the server with the domain so the system can be recognized on the Internet.

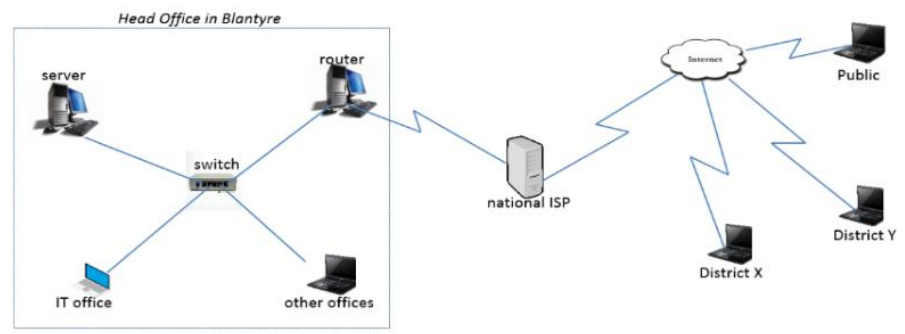

Fig. 1: Implementation of IS for Organisation A (Note: Images are from Microsoft Office)

The participatory design was applied. During the requirements analysis, one meeting was organised with district managers and users to redesign data collection forms to accommodate new requirements. They were also introduced to the innovative idea of computerising IS. During the system development, the developers worked closely with managers at the Head Office whom each finished function was demonstrated for their feedback. The system was tested in three district offices using live data and modifications were done accordingly. During the system implementation, another meeting with district managers was conductedin which the finished product was demonstrated for the feedback. Another meeting was organised for other stakeholders for their feedback and suggestions on how to integrate the system with other IS of collaborating partners for easy exchange of data.

\subsection{Organisation B (NGO)}

This case involves the development of M\&E system by local consultantsfor monitoring its community-based programs in some districts in Malawi. The IS development was initiated by the organisation itself in 2014. The development process involved four main stages: user requirements analysis, design and development, testing, and implementation. During the user requirements analysis, developers interacted with user representatives at their workplace to 
understand theirrequirements. In the next two stages, the developers designed and developed the system with little consultation with users. User representatives were later involved in the system testing and implementation in which they provided feedback and got training.During the development, developers and users were in different cities and they were mainly communicating through phone calls and emails. "We utilised phone calls and emails particularly during system design and development because it was expensive for us to travel to Lilongwe from Blantyre in terms time and finances. We only met where there was something to demonstrate that require instant feedback and clarification" - one developer elaborated. The distance between Blantyre and Lilongwe is about 320 kilometres.

The architecture of M\&E system is in two-tier including data layer and business logic (plus interfaces). The developers used PHP and MySQL for the business logic and database respectively. The system is hosted in South Africa on Windows server and users are able to access it in Malawi through the Internet. Developers were given temporary access to the server for uploading and testing the software. The system is only for few staff members who are responsible for M\&E services. The user can also download reports in the form of spreadsheet, word format and pdf.

In this case, it has been learned that one consultant did the requirements analysis and another consultant did the design and development. In the first phase, the later was not present. This practice brought some confusion in the systemdevelopment, which delayed the project by some months. "Since I was not involved in the analysis, it took me time to understand the requirements because they were poorly documented" - the later emphasised. One issue was that some functions of the system were not meeting user requirements and hence, they were redone; During the systemdevelopment, much refinement of requirements was done.

\subsection{Organisation C (six SMEs)}

The accounting and finance package for SMEs has been developed with the understanding that most SMEs cannot afford to buy and maintain commercial accounting packages which are relatively expensive. The commercial accounting packages are also sophisticated and they require high technical knowledge in accounting and technology, which most SMEs do not have. They are also difficult to customise to meet specific business needs of SMEs. Although there is the option of free and open source accounting packages, these packages require some sort of customisation that needs technical expertise. Most SMEs in Malawi have no capacity to engage the technical expertise to manage their computerised accounting systems. Hence, majority use Microsoft Excel to manage their accounts and finances. For instance, out of six visited SMEs, four were using Excel and two had manual accounting systems.

The current version of accounting and finance package focuses on the finance, business accounts, and business planning. It supports business operations including management of customers, suppliers, products, banking, transportation and logistics, and planning. The package also involves transactions on sales, purchases, debtors, expenses, fixed assets and payments. In terms of the bookkeeping, the package has modules for invoicing and cash accounts. Apart from the main accounting modules, the package also has security modules, which allows only registered users with right credentials and every transaction is logged (audit trail). It generates general standard reports that are relevant to SMEs. However, the reports are not customisable but they are presented in Excel that can allow users to process data further when a need arises. 
A local developer initiated the development of the accounting and finance package in 2013. By 2015, twenty-four SMEs were using the package mainly in Blantyre, Lilongwe and Zomba cities. It is a standalone application software developed in Visual Basic .NET with support of Microsoft Access for the database and Excel for reporting. "We developed the standard package to run in Windows environments because during our analysis stage we observed that most SMEs were using Windows "- one developer emphasised.

Four SMEs moved from Excel to the computerised accounting system while two moved from the manual to computerised accounting system. One SME has an accountant and five SMEs have managers as users of the package. All six visited SMEs indicated that they were given basic training separately on how to operate the system. If they have any operational problems, they are supported well either through the phone or physical visits. "We have at least one representative in each city to support our customers." - one developer commented.

\section{Discussions}

The findings have been presented in two views: 'application' and 'developer' domains. In the 'application' domain, ICT can be considered as tools that support the operations and users require adequate knowledge and skills [16]. In the 'developer' domain, the focus is on the development of IS, which is usually guided by needs and demands of the 'application' domain [16]. Hence, both users and developers need to interact during the IS development.

\subsection{Organisation perspective}

Organisation can be taken as a governance structure that is established to manage human interactions and its rules, regulations, policies, strategies and procedures among others form the basis for guiding activities of the organisation [17]. The interest was on strategies adopted in the IS development and parts of governance structures supported by IS solutions (see Table 2).

Table 2.Organisation dimension in three cases

\begin{tabular}{llll}
\hline Characteristics & Organisation A & Organisation B & Organisation C \\
\hline Governance & & & \\
- Users & - National \& district levels & - M\&E at National level & - Manager \& few staff \\
- ICT Unit & - Yes & - ICT Officer & - No \\
- Policies \& & - No & - No & - No \\
standards & & & \\
$\begin{array}{c}\text { Development } \\
\text { - Initiation }\end{array}$ & - By user & - By user & - By developer \\
- Planning & - Yes & - Yes & - No \\
- Approach & - Incremental, in-house, & - Waterfall, contracting & - Waterfall,off-the- \\
& staff \& volunteer & out, consultants & shelf \\
- Management & - Yes & - Yes & - Yes \\
commitment & & & \\
\hline
\end{tabular}

In all three cases, the IS solutions have been implemented to enhance the work processes for particular offices. For the successful use of the IS solutions, these offices need the continuing technical and organisational support. It has been noted that different organisations have different approaches to provide such support. From Table 2, Organisation A and Organisation B have ICT officers, as part of the employment, to provide necessary technical 
support while Organisation $\mathrm{C}$ gets the external technical support. Unfortunately, in all three cases, there are no policies or standards to guide the implementation and use of IS solutions.

In IS development, individuals or group of people usually initiate the process. In the context of 'application' and 'developer' domains, the IS development project is initiated by either the user organisation or developer. If the initiation is from the user organisation, it is the organisationitself, which decides to implement a new IS solution or it is the decision of the sector or industry in which the user organisation belongs or participates. An example can be the public institution; the government can decide to introduce IS solutions across ministries, departments or agencies. However, Organisation A has decided itself to implement the IS solution. Organisation B is part of the international NGO having offices in different countries and it is a policy of the 'global' management to automate M\&E systems in individual country offices. However, the individual country management makes decisions on how and when to implement IS solutions. In the case of Organisation $\mathrm{C}$, the decision of implementing IS solution was made by the developers after observing some challenges being faced by SMEs in accounting and finance. It has been observed that where decisions of implementing IS solutions are made by the user organisations, plans exist (see Table 2).

\subsection{Human perspective}

As mentioned earlier, IS cannot survive without people [3] including those who enter data into the system and also who receive and use information from the system. This explanation seems to target the 'application' domain whose focus is on users of IS. However, from the perspective of IS development, groups of people involved are beyond the users. As shown in Table 3 for three cases, the IS development needs also developers because it involves various activities (i.e. investigation, analysis, design, programming, testing, implementation, operation and maintenance), which require the involvement of both developers and users. IS designs include developers and users with their own unique group and individual perspectives, and they may need to collaboratively determine how the system can be implemented, satisfying developers' and users' constraints [18].

Table 3.Human dimension in three cases

\begin{tabular}{llll}
\hline Characteristics & Organisation A & Organisation B & Organisation C \\
\hline Developers & - Volunteer \& IT officers & - Individual consultants & - Individuals \\
Users & - All officers \& the public & - One office & - Individuals \\
Support & - Continous & - Continous & - Continous \\
Training & - Yes & - Yes & - Yes \\
Documentation & - Operational and technical manuals & - Operational manual & - Operational manual \\
\hline
\end{tabular}

From the findings, it has been observed that there is another group of people who are called implementers. These groups of people (developers, implementers, and users) have been discussed with relation three cases. Developers are taken as individuals who are responsible for designing and developing software solutions for IS, who are not only restricted to programming, but also perform other duties such as analysis, implementation, training and support. Developers belong to the 'developer' domain and can come from within the client organisation as part of users or outside, for example, as consultants or volunteers. In the 'application' domain, there are users who are individuals using IS to perform various tasks. Those users can be grouped into two: expert users and novices [19]. Expert users are familiar and experienced with the system while novices are end-users who are not much familiar with the system and hence they need support from expert users and/or implementers [41]. Implementers are individuals who are only response for performing activities in the 
implementation of IS. Implementers can also be developers and/or expert users. Figure 5 illustrates interactions between developers, implementers, expert users and novices.

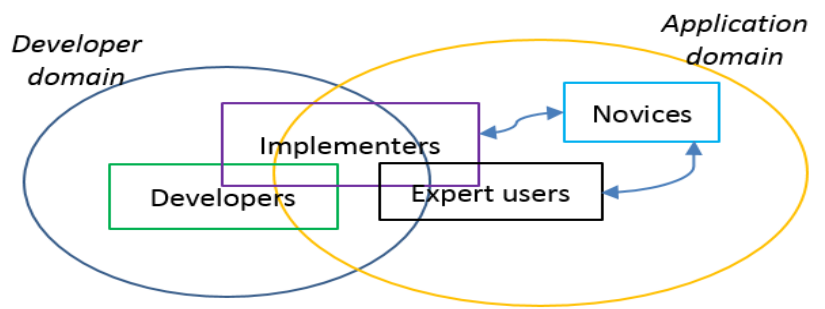

Fig 5: Interactions between developers, implementers and users

All three cases fit in the model in Figure 5. In Organisation A, ICT unit plays three roles as developers, implementers and expert users. ICT officers, as developers, participated in analysis, design, programming and test of the system together with the volunteer. They were involved in the implementation of the system. Their involvement in the development and implementation activities has given ICT officers necessary knowledge and skills; making them expert users who provide needed technical support to other users - novices. The volunteer as an implementer also interacts with novices. In Organisation B, the case is slightly different from that in Organisation A. Although Organisation B has an ICT officer, it was only involved in the implementation stage, but not in the development stage. The IS solution was totally developed by the consultants. In this case, the ICT officer is both expert user and implementer. Technical knowledge was transferred to the ICT officer through training, manuals and participation in the implementation for enabling the officer to provide necessary support to $\mathrm{M} \& \mathrm{E}$ officers as novices. In the case of Organisation $\mathrm{C}$, developers and implementers were from outside the 'application' domain. In all six visited SMEs, there are no expert users. Novices get support from the implementers. Developers of the package have hired some individuals who are trained to implement and provide support to SMEs, as users of the package. Since they are implementers, the hired individuals provide necessary support to novices.

Generally, people, who are involved in the IS development as developers, implementers or users, need certain levels of competencies, knowledge and abilities to perform labour. Chikumba and Msendema [20] refer to the stock of these elements as the human capital, which increases through education, training and experience. It has been observed that developers in all three cases acquired their knowledge and skills through education system. They are university graduates who have learnt programming and systems analysis and design during their university studies. In the case of users, they obtained the required knowledge and skills through training, manuals and interactions with developers and/or implementers.

\subsection{Technology perspective}

Technology as discussed provides the platform on which the ISs run. The Systems are built on these technologies:Hardware, Operating Systems, Network (Servers, client machines, and mode of connection.) Acrosss the case organistions, acqusion of the desired technology and expertise has been observed as the core challenge. As highlited in Table 4 open source technologies have proved to be a solution to enterprise commercial technologies and simple implimentation architecture which dont require high level exprtise to develop and support have been the option across the organisatins under study. 
The Internet and World Wide Web have also enabled these organisation realise their ambition of automation and increased access and sharing of operational resources. The advantage of these is that the organisations do not necessarily need to have own advanced technologies as they do benefit from service providers' infrastructre like ISPs at a small subscription[4.1].

It has been observed that basic computers, open source engines like the PHP and MySQL Server, Operating Systsems like Linux and Windows (which normaly comes pre-installed in pcs) have been the enablers of adaptation of CIT in resource contrained environment because they tend to require basic training and low investment cost but still propelling the respecitve organisations operations.

The only concern however is that as these organisations mostly rely on volunter expertise and individual initiative in the development and support of these technologies and systems, they risk huge business disturbances should these individuals get engaged in other businesses and move away. On the other hand lack of full technical documentation may prove a problem towards maintenance and growth of these systems. [21]. There is a need to address these to ensure continued reliable information systems in such constrained environments.

Table 4.Technology dimension in three cases

\begin{tabular}{llll}
\hline Characteristics & Organisation A & Organisation B & Organisation C \\
\hline Architecture & - Web-based IS, two tier, mix of & - Web-based IS, two tier, & - Standalone IS, two tier, \\
& technologies & mix of technologies & mix of technologies \\
Programming & - PHP \& MySQL & - PHP \& MySQL & - VB NET \& Access \\
OS & - Linux server & - Windows server & - Windows client \\
Hosting & - Hosted locally & - Hosted outside & - Copy for each user \\
Hardware & - PC as server, PCs for clients, & - PCs for clients, printers & - PCs for clients, printers \\
& printers for users & for users & for users \\
Accessibility & - Internet \& LAN & - Internet & - Individual PCs \\
Other tools & - Excel, Word, PDF & - Excel, Word, PDF & - Excel \\
\hline
\end{tabular}

\section{Conclusion}

In this paper we have systematically analysed the potential of adapting information systems in resource constrained environment. The findings have supported the hypotheses that "organisations with limited resources can still make the most of ICT and that resource contraints motivate efficiency and creativity within the organisations". The study however did not go further to find the best way to mitigate the challenges that come with over reliance on volunteers and open source technologies as the systems grow.

The findings of the study can help individuals, SMEs and organisation with limited resource learn the best ways they can capitalise on what they have, the open source technologies and volunteers to develop and manage informations systems and improve their businesses processes. Risks associated with these have not been studied in detail and future research can consoidate on such areas so that a reliable model can be developed to guide the development and implementation of Information Systems in resource constrained environments. 


\section{References}

[1] UNCTAD, Information Economy Report 2012: The Software Industry and Developing Countries, United Nations, New York, 2012.

[2] A. Mursu, H. A. Soriyan, K. Olufokunbi, and M. Korpela, "Information Systems Development in a Developing Country: Theoretical Analysis of Special Requirements in Nigeria and Africa," in 33rd Hawaii International Conference on System Sciences, 2000.

[3] J. Galbreath, "Which resources matter the most to firm success? An exploratory study of resource-based theory," Technovation, vol. 25, no. 9, pp. 979-987, September 2005, 2005.

[4] A. Naber, and R. Kolisch, "MIP models for resource-constrained project scheduling with flexible resource profiles," European Journal of Operational Research, vol. 239, pp. 335-348, 2014.

[5] R. Suttle. "The Advantages and Disadvantages of Resource Constrained Projects,", 20 July, 2017; http://smallbusiness.chron.com/advantages-disadvantages-resourceconstrained-projects-33592.html.

[6] I. Apulu, and A. Latham, "Drivers for Information and Communication Technology Adoption: A Case Study of Nigerian Small and Medium Sized Enterprises," International Journal of Business and Management, vol. 6, no. 5, pp. 51-60, 2011.

[7] S. Sawyer, and M. Tyworth, "Social informatics: Principles, theory, and practice," in IFIP International Conference on Human Choice and Computers, US, 2006, pp. 49 62.

[8] G. A. Macueve, "Drugs Logistics Management Information System in Mozambique: Challenges and Opportunities," Thesis, Department of Informatics, University of Oslo, Norway, 2003.

[9] J. M. Twati, "Societal and Organisational Culture and the Adoption of Management Information Systems in Arab Countries," Thesis, Griffith University, 2006.

[10] P. Isaias, and T. Issa, High Level Models and Methodologies for Information Systems, New York: Springer Science+Business Media 2015.

[11] D. Boddy, A. Boonstra, and G. Kennedy, Managing Information Systems: An Organisational Perspective, 2nd ed.: Financial Times Prentice Hall, 2005.

[12] F. Mukama, H. C. Kimaro, and J. Gregory, "Organization Culture and its Impact in Information Systems Development and Implementation: A case study from the health information system in Tanzania."

[13] D. Kunda, "A social-technical approach to selecting software supporting COTSBased Systems,” Thesis, Computer Science, University of York, UK, 2001.

[14] E. Turban, R. K. J. Rainer, and R. Potter, Introduction to Information Technology, 2nd ed.: John Wiley \& Sons, Inc., 2005.

[15] J. Green, K. Willis, E. Hughes, R. Small, N. Welch, L. Gibbs, and J. Daly, "Generating best evidence from qualitative research: the role of data analysis," Australian and New Zealand Journal of Public Health, vol. 31, no. 6, pp. 545-550, 2007.

[16] J. Campbell, and M. Shin, Essentials to Geographic Information Systems, New York: Flat World Knowledge, Inc., 2011.

[17] N. Nemarundwe, "Institutional collaboration and shared learning for forest management in Chivi District, Zimbabwe," Social Learning in Community Forestry Management: Linking Concepts and Practices, E. Wollenberg, D. Edmunds, L. 
Buck, J. Fox and S. Brodt, eds., pp. 85 - 108 Bogor: Center for International Forestry Research, 2002.

[18] D. H. Sonnenwald, "Contested Collaboration: A Descriptive Model of Intergroup Communication in Information System Design," Information Processing \& Management, vol. 31, no. 6, pp. 859-877, 1995.

[19] P. A. Chikumba, and G. Chisakasa, "GIS Implementation for Health Management in Malawi: Opportunities to Share Knowledge between Implementers and Users," American Journal of Geographic Information System, vol. 6, no. 4, 2017.

[20] P. A. Chikumba, and M. Msendema, "Computer Education and Training: Human Capacity Building for e-Government in Malawi," AFRICOMM 2014. LNICST, A. Nungu, B. Pehrson and J. Sansa-Otim, eds., pp. 180-188, Heidelberg: Springer, 2015.

[21] M.Heron, V.L Hanson and I. Ricketts," Open source and accessibility: advantages and limitations," Journal of Interaction Science, Vol. 1, no 2, 2013 